\title{
Un régleur électrique de vitesse
}

\section{Electric Governor}

\author{
PAR M. CUENOD (1) \\ ING. Dipl. E.P.F., ZURICH
}

English synopsis p. 95

\section{I. - INTRoduction}

Le problème du réglage de la vitesse des turbines date de la création des premières turbines. En effet, en réglant leur couple moteur de façon à maintenir leur vitesse constante, on ajuste ainsi la puissance à produire par le groupe à la puissance demandée par la consommation.

Les constructeurs de turbines vouèrent une attention toute spéciale à la réalisation de régleurs tachymétriques mécaniques qui ont atteint aujourd'hui un degré très élevé de précision, de sensibilité, de rapidité, et qui satisfont actuellement dans la majorité des cas à des conditions de marche très exigeantes.

Cependant, l'entraînement mécanique du régleur des turbines de grande puissance peut présenter parfois certaines difficultés, lorsque, du fait des dimensions du groupe, le régulateur se trouve éloigné de l'axe de la turbine:

Pour éviter une transmission mécanique à l'aide de courroies et d'engrenages qui sont utilisés sans difficulté pour les groupes de faible puissance, on a été conduit à se servir d'une transmission électrique. Le régleur est alors entrainé par un moteur qui, dans le cas le plus simple, est alimenté par un transformateur de faible puissance, placé aux bornes de l'alternateur. Ce mode d'alimentation a l'avantage d'être très simple et bon marché, mais présente l'inconvénient de soumettre l'entrainement du régleur à toutes les vicissitudes auxquelles la tension du réseau peut être exposée, par suite de la possibilité de court-circuits, qui risquent de faire baisser passagèrement la tension aux bornes du

(1) Ingénieur à l'Ofnico, 2, rue de la Tertasse, Genève (Suisse). générateur, de faire « décrocher» le moteur du régleur et d'ouvrir la turbine inopportunément. Pour obvier à cet inconvénient, il est nécessaire de prévoir un relais de surveillance pour fermer la turbine en cas de manque de tension. Lorsque, par suite d'un court-circuit dans une partie éloignée du réseau la tension ne disparaît pas complètement mais diminue fortement, le fonctionnement du relais est indéterminé; il peut être amené à vibrer c'est-à-dire à s'ouvrir et à se fermer successivement selon de faibles variations de la tension.

Pour cette raison, lorsque l'on adopte un régulateur à transmission électrique, on prévoit généralement pour l'alimentation du moteur du régleur, un petit générateur ad hoc appelé « générateur pilote $»$, placé, dans la majorité des cas, sur l'arbre du générateur principal.

Le réglage de vitesse se trouve ainsi à l'abri des perturbations qui peuvent intervenir sur le réseau.

Toutefois, par suite de l'inertie des masses mises en mouvement et des champs magnétiques, les variations de la vitesse du moteur du régleur ne suivent pas immédiatement les variations de la vitesse de l'alternateur principal. Ce « décalage » diminue considérablement l'efficacité des excellentes qualités du régleur tachymétrique ou accéléro-tachymétrique et risque, dans certains cas, heureusement peu fréquents, de compromettre la stabilité même du réglage.

D'autre part, le prix et l'encombrement du générateur pilote sont relativement élevés, en particulier s'il doit être monté sur l'arbre principal d'un groupe lent avec turbine Kaplan, du fait que l'arbre d'une telle turbine est creux, et par suite du grand nombre de pôles dont il faut pourvoir dans ce cas le générateur pilote. Ainsi pour 
$75 \mathrm{t} / \mathrm{min}$. il faut 80 pòles afin d'obtenir une fréquence de $50 \mathrm{~Hz}$.

On peut remplacer le dit générateur pilote en exécutant une excitatrice pilote avec des bagues pour collecter le courant alternatif demandé. Mais, dans ce cas également, se présente la difficulté de la nécessité d'un grand nombre de pôles pour un groupe lent. Le démarrage qui peut se faire avec excitation par hatterie, n'offre plus la même élégance que dans le cas d'un alternateur pilote séparé.

L'électricien qui voit de près les différents inconvénients de la transmission électrique avec un moteur pour entraîner le régleur propose d'y parer en remplaçant le régleur tachymétrique, son moteur et le relais de surveillance, par un régleur électrique qui permet d'obtenir directement un couple de commande en fonction de la fréquence du courant électrique.

Ce régleur électrique a la propricté intéressante de fermer automatiquement la turbine lorsque la tension disparait; d'autre part, il requiert une puissance bien inférieure à celle requise par le moteur d'un régleur, aussi il permet de réduire considérablement les dimensions du générateur pilote.

Nous nous proposons dans le présent exposé de donner le principe d'un tel régleur électrique ainsi que l'a réalisé la Maison Brown-Boveri à Baden (Suisse). Nous en décrirons le fonctionnement et indiquerons quelques résultats d'exploitation.

\section{II. - PRINCIPE dU RÉglevr Ǵlectrigue DE VITESSE}

On sait depuis longtemps mesurer une fré-

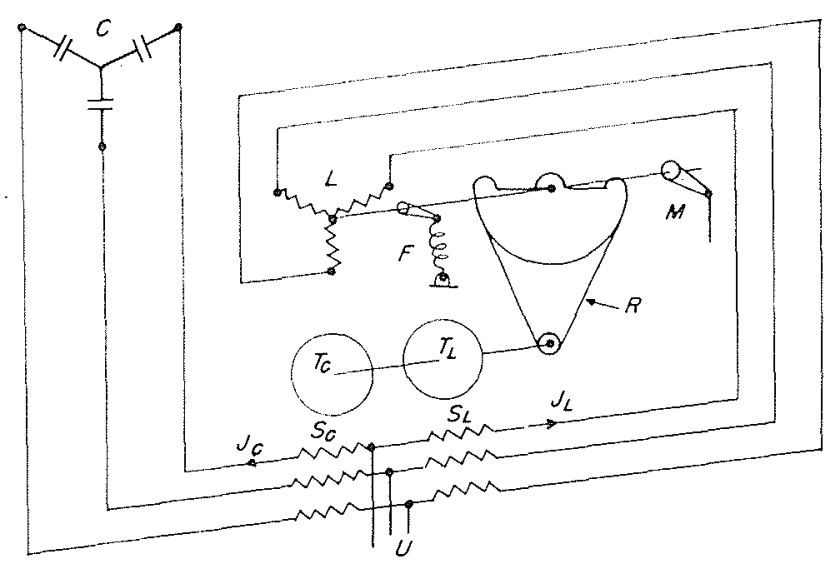

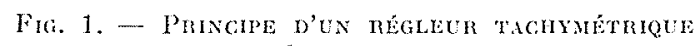
ELECTRIQUE

quence électrique à l'aide d'un système mobile dont le couple est proportionnel à l'écart de fréquence; pour obtenir un régleur de vitesse par ce moyen, il fallait que le couple obtenu se-
Ion le principe représenté par la figure 1 fût suffisant pour attaquer le distributeur du servo-moteur de la turbine.

Le dispositif de mesure alimenté par la tension $U$ comporte 2 tambours $T_{C}$ et $T_{\mathrm{T}}$ fixés sur un même axe et pivotant chacun dans un stator triphasé. L'enroulement du stator $S_{C}$ est branché en série avec le condensateur triphasé C. L'enroulement du stator $S_{L}$ est branché en série avec l'inductance variable triphasée $L$; l'axe du rotor de cette inductance est accouplé à l'axe du système mobile par l'intermédiaire du ruban d'accouplement $R$ selon un rapport de transmission donné. Le choix des valeurs de capacité, d'inductance et de l'enroulement des stators du dispositif de mesure est tel que, pour la fréquence nominale, les couples auxquels les tambours $\mathrm{T}_{\mathrm{C}}$ et $\mathrm{T}_{\mathrm{T}}$ sont soumis par suite de l'eflet de Ferraris soient egaux et de sens opposés.

Le système mobile du régleur est alors en repos dans sa position médiane. Admettons que la fréquence monte, le courant dans le circuit de mesure capacitif va augmenter tandis que le courant dans le circuit de mesure inductif va diminuer. Le couple auquel le tambour ' $\mathrm{T}_{\mathrm{C}}$ est soumis l'emporte sur celui auquel le tambour $\mathrm{T}_{\mathrm{r}}$, est soumis.

Le système de mesure va tourner autour de son axe et entrainer le rotor de l'inductance $\mathrm{L}$. de facon à diminuer la valeur de cette inductance jusqu'à ce que le courant dans le chrcuit de mesure inductif augmente suffisamment pour rétablir l'équilibre des couples. Le régleur tachymétrique électrique a donc un caractère statique. Pour chaque fréquence -- partant pour chaque vitesse du sroupe - son systeme mobile adopte une position bien déterminée. Sa sensibilité est excellente ainsi que cela ressort des considérations suivantes.

Le couple auquel est soumis un système Ferraris est proportionnel au carré du courant qui en est la cause. Le courant du circuit capacitif est proportionnel à la fréquence, celui du circuit inductif est inversément proportionnel à la fréquence. Nous obtenons en fonction de la fréquence les valeurs relatives du couple suivant :

couple du système capacitif :

$$
\frac{\mathrm{M}_{\mathrm{C}}}{\mathrm{M}_{\mathrm{O}}}=(1+y)^{2} \cong 1+2 y
$$

couple du système inductif :

$$
\frac{\mathrm{M}_{\mathrm{L}}}{\mathrm{M}_{0}}=\frac{1}{(1+y)^{2}} \cong 1-2 y
$$

avec $\nu=$ écart relatif de fréquence $=\frac{\Delta f}{f_{0}}(\leqslant 1)$

Nous pouvons ainsi déterminer l'écart relatif 
du couple qui résulterait d'un écart de fréquence unitaire si l'on bloquait le système mobile.

$$
\begin{aligned}
& =\frac{\Delta \mathrm{M}}{\mathrm{M}_{0}}=\frac{\mathrm{M}_{\mathrm{C}}-\mathrm{M}_{\mathrm{L}}}{\mathrm{M}_{0}} \\
& =(1-2 y)-(1-2 y)=4 y \\
& \text { gradient } \frac{\mu}{y}=4
\end{aligned}
$$

A titre de comparaison, nous déterminons le gradient du régleur tachymétrique à force centrifuge (proportionnelle au carré de la vitesse) :

$$
\begin{aligned}
\frac{F}{F_{0}} & =(1+\nu)^{2} \cong 1+2, \\
0 & =\frac{\Delta F}{F_{0}}=\frac{F-F_{0}}{F_{0}}=2, \\
\text { gradient } & =\frac{t}{y}=2
\end{aligned}
$$

soit la moitié du gradient du régleur électrique.

Ces considérations sont confirmées par l'expérience qui a prouvé que l'insensibilité du régleur tachymétrique électrique était inférieure à $\pm 0,3: 1.000$.

Le levier M, qui attaque le distributeur du

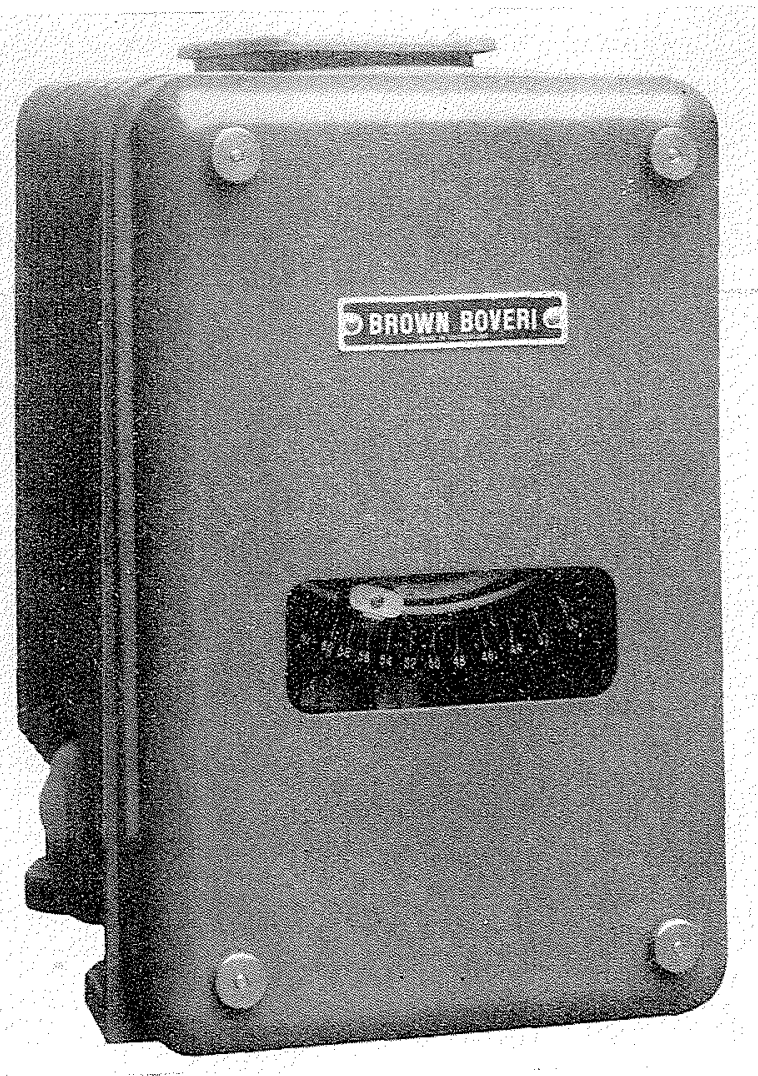

Frg. 2. - Vue d'Un Regleur tachraétrioue ílectroue

A galche : Face antéricure avec levier indiquant la position du système mobile du régleur a l'aide d'une échelle graduécen $\mathrm{Hz}$. servomoteur du dispositif de réglage de la turbine, est fixé sur l'arbre qui porte le rotor de la self L; grâce au rapport de transmission du r'uban d'accouplement, il est possible d'obtenir un couple de $36 \mathrm{kgcm}$ pour une variation de fréquence de $1 \mathrm{~Hz}$ (le rotor élant bloqué).

$\mathrm{Vu}$ que les deux circuits de mesure sonl branchés à la même tension, les deux couples antagonistes varient simultanément en cas de variation de tension; c'est la raison pour laquelle la mesure de la fréquence est, dans un large domaine, indépendante de la tension. Une réduction de la tension n'a une influence que sur la sensibilité du régleur; si la tension disparait complètement, les deux couples antagonistes s'annulent, le ressort de fermeture $F$ fait en sorte que le régleur commande alors la fermeture du vannage de la turbine. L'influence de ce ressort est négligeable à la tension nominale; on en tient compte d'ailleurs dans l'étalonnage du régleur.

Les photos de la figure 2 donnent une vue d'un régleur tachymétrique électrique tel que le réalise la Maison Brown, Boveri et Cie à Baden (Suisse). La photo 2 a donne une vue de la face antérieure de ce régleur, la photo $2 b$ de la face postérieure avec l'arbre qui permet d'attaquer

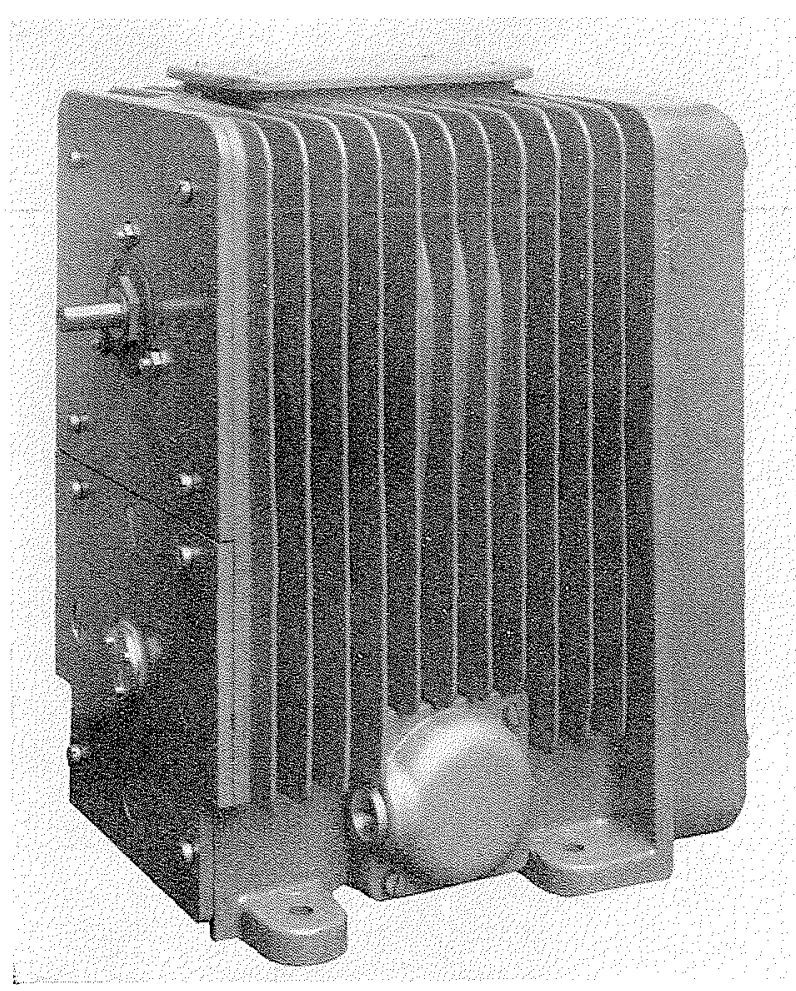

A droite: Face postérieure avec l'arbre sur lequel agit le couple de commande qui permet d'attaquer le dispositif de réglage de la turbine. 


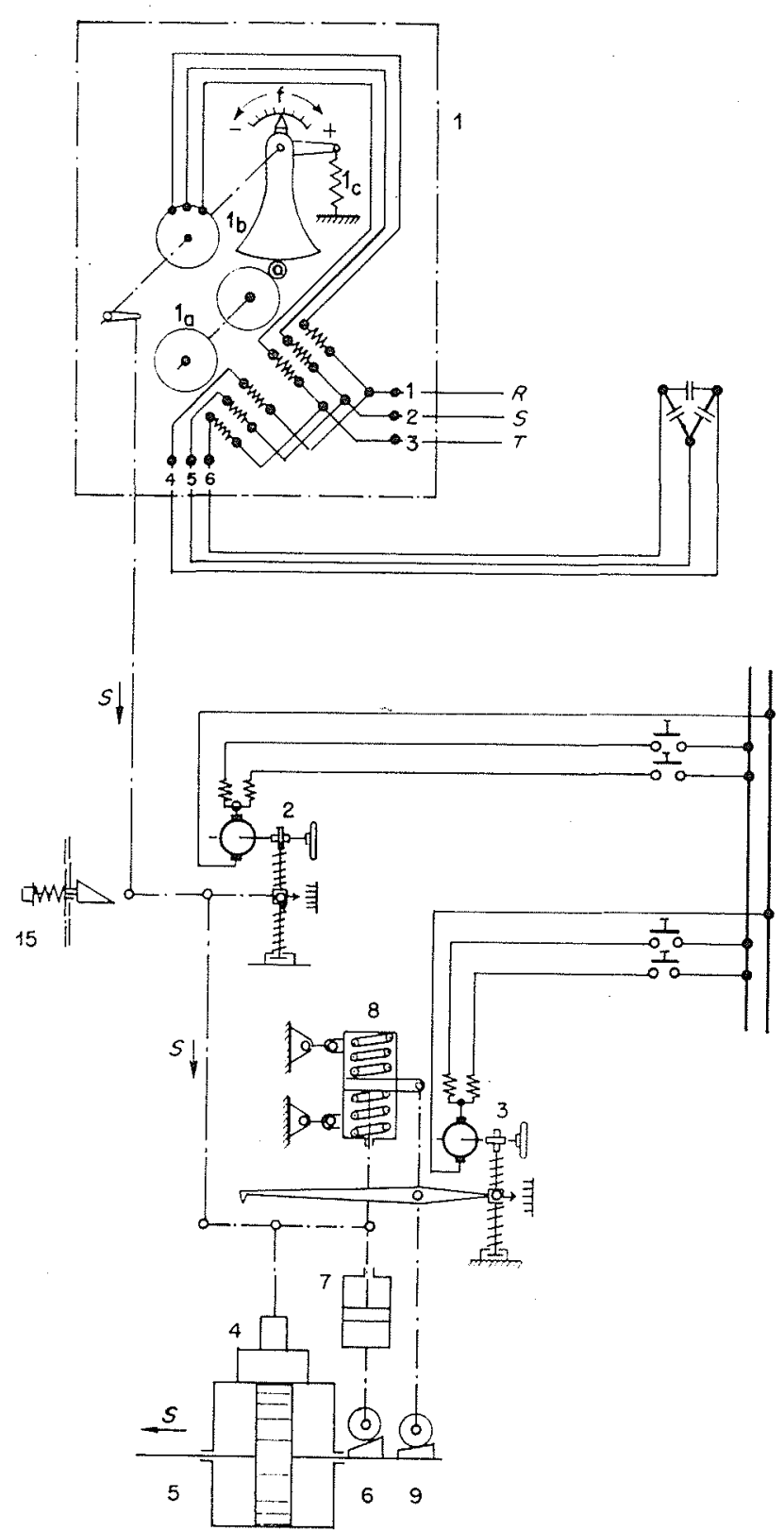

Fig. 3. - A. Réguláteun

1. Régleur électrique de vitesse type LPS :

1a Détecteur tachymétrique électrique,

1b Régulateur d'induction à rotor mobile,

1c Ressort de fermeture.

2. Dispositif de changement de vitesse avec moteur pour commande à distance.

3. Dispositif de limitation d'ouverture avec moteur pour commande à distance.

4. Tiroir du distributeur. B. AMPLIFICATEU

5. Servomoteur.

C. Dispositif de stabllisation

6. Statisme momentané

7. Amortisseur.

8. Ressorts de rappel.

9. Statisme permanent.

15. Dispositif de démarrage à main.

S. Mouvement de fermeture. le dispositif de réglage de la turbine. Le couvercle du régleur, que l'on peut enlever facilement, est muni d'une fenêtre qui permet de lire directement la position du système mobile sur une échelle graduée en $\mathrm{Hz}$. Cet appareil est d'une construction très robuste. Il mesure environ $40 \mathrm{~cm}$ de haut. Système de mesure et inductance sont montés dans le même boîtier; par contre, les condensateurs doivent être montés à part.

\section{III. - Fonctionnement du RÉglevr Électrigue DE VITESSE}

La figure 3 donne une représentation schématique d'un dispositif de réglage d'une turbine hydraulique avec régleur tachymétrique électrique.

Le tiroir de distribution 4 du servomoteur 5 est attaqué d'une part par le régleur électrique 1. d'autre part par le dispositif du changement de vitesse 2 et le dispositif d'asservissement. La came 6 détermine le statisme passager par l'intermédiaire de l'amortisseur 7 et du ressort 8 . La came 9 détermine le statisme permanent en déplaçant le point d'appui du ressort 8. Lorsqu'à la suite d'une variation de la fréquence, le pislon du servomoteur 5 se déplace, la came 6 agit par l'intermédiaire de l'amortisseur 7 sur le tiroir de distribution en sens inverse de celui de la correction du régleur. Ce faisant, le ressort 8 est tendu; il se détend ensuite peu à peu par le jeu du piston de l'amortisseur 7 et retrouve sa position médiane. Le servomoteur continue sa course jusqu'à ce que, grâce à la came 9 du statisme permanent, la course occasionnée par l'action du régleur soit compensée par celle occasionnée par l'asservissement permanent, et que le tiroir de distribution ait ainsi retrouvé sa position d'équilibre.

Le dispositif du changement de vitesse peut être commandé soit à main, soit à distance, par l'action d'un moteur. La charge de la turbine est limitée par le dispositif de limitation d'ouverture 3 qui peut être commandé à distance à l'aide d'un moteur. La mise en marche du groupe s'exécule à l'aide du dispositif de démarrage à main 15 .

Ces trois dispositifs font partie du régulateur normal de la turbine. Ils ne sont modifiés en rien par la présence du régleur tachymétrique

Fig. 4. - Vue dU DispositTF DE RÉGLage D'UN GROUPE HYDTO-HLECTRIQVE AVEG TURBINE KaPLAN ET RÉgulateur PRMAIRE

Cn reconnait au premier plan le dispositif de réglage de la turbine munie d'un régleur tachymétrique électrique. 


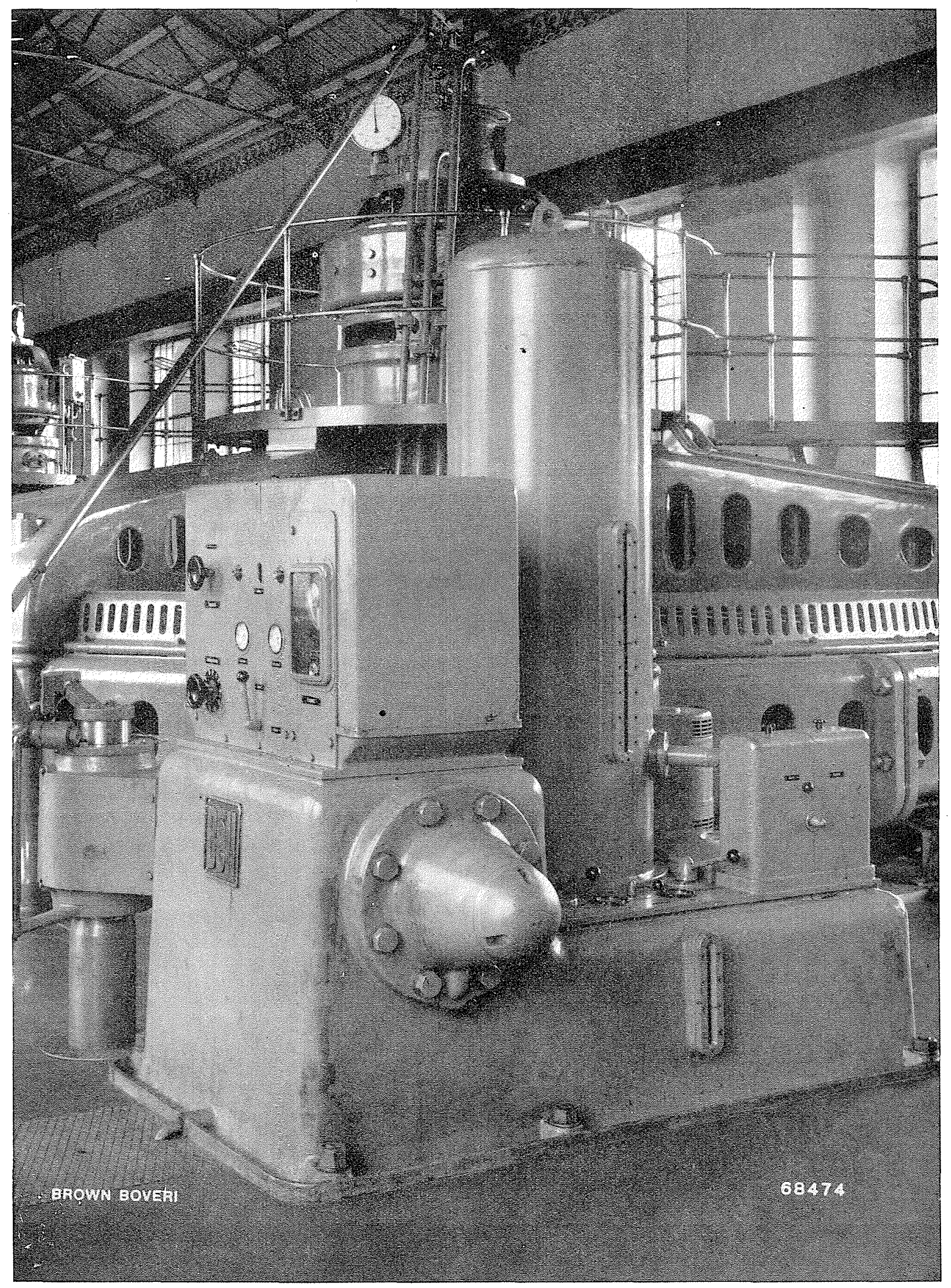


mécanique qui s'adapte ainsi facilement au dispositif de rẻglage habituel d'une turbine hy draulique.

La photo de la figure 4 donne la vue du dispositif de réglage d'une turbine Kaplan dotée d'un régleur électrique de vitesse.

a)

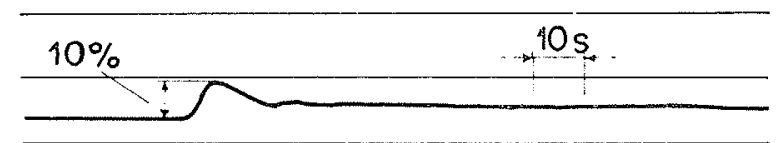

b).

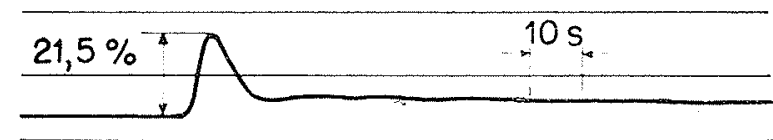

Fig. 5. - Diagname de vitesse a la suite des variations brusques de charge d'un groupe commandé par un régleur tachymétrique électrique. Turbine Kaplan : 22.000 kVA. $75 \mathrm{t} / \mathrm{m}$

a) Décharge de $9.600 \mathrm{~kW}$ survitesse max : $10 \%$.

b) Décharge de $16.000 \mathrm{~kW}$ survitesse max : $21,5 \%$

La figure 5 permet de se faire une idée du comportement du régleur à la suite d'une brusque variation de la charge de la turbine. Ce cliché donne le diagramme de déclenchement pour une turbine Kaplan d'un groupe de $22.000 \mathrm{kVA}$.

Nous royons qu'apres une survitesse passagère restant dans les limites admissibles, la vitesse retrouve rapidement sa valeur de consigne, pratiquement sans oscillation de réglage.

L'excellence de ces résultats rend hommage aux propriétés tant du dispositif de réslage de la turbine que du régleur électrique.

\section{IV. - Conchuston}

Le régleur électrique de vitesse apporte une contribution intéressante au problème du réglage de vitesse des turbines hydrauliques. Son montage est commode puisqu'il ne nécessite attcun dispositif d'entraînement mécanique. Il no change en rien le dispositif de réglage de la turbine. Il ne nécessite aucun asservissement supplémentaire mais vient simplement en licu et place du régleur tachymétrique mécanique. Sa sensibilité est très élevée grâce à la caractéristique favorable de son couple en fonction de la fréquence.

L'inertie mécanique et magnétique du dismositif de mesure est relativement très faible par rapport à celle de l'ensemble du circuit de réglage; la bonne caractéristique dynamique du régleur électrique est prouvée par son action favorable tant sur la stabilité du groupe que sur son comportement à la suite d'une décharge brusque.

La consommation du régleur électrique est relativement très faible (environ $150 \mathrm{VA}$ à $\cos 0$ $=1$ ). Il peut être alimenté à volonté soit par le générateur principal ou soit par un alternateur pilote. Dans ce dernier cas, la consommation réduite du régleur électrique permet de réduire considérablement les dimensions du générateur pilote, ce qui constitue une économie appréciable. Il est possible de prévoir un petit alternateur à pôles permanents, monté par exemple sur le train d'engrenage d'une pompe de la turbine. On évite ainsi le montage d'une machine accessoire sur l'arbre principal du groupe qui porte souvent déjà 1 ou 2 excitatrices.

Le régleur tachymétrique électrique fonctionne d'une facon satisfaisante à une tension réduite jusqu'à 25 à $30 \%$ de la tension nominale. En cas de disparition de la tension, ce régleur est concu de facon à fermer automatiquement louverture de la turbine. Ces deux propriétés garantissent une grande sécurité de marche, et sont avantageuses en particulier lorsque l'on se propose d'alimenter le régleur électrique aux bornes du générateur, ce qui permet d'éliminer l'influence des oscillations du rotor sans qu'il $y$ ait à redouter les conséquences que les perturbations de la tension peuvent aroir sur la marche du régleur tachymétrique mécanique, entrainc par un moteur.

II ressort de ces considérations que lorsqu'une transmission électrique est envisagée pour la commande d'une turbine, certains des avantages que présente le régleur tachymétrique électrique lui permettent de rendre un service indéniable. Son utilisation est un exemple de l'entente étroite qui est toujours plus nécessaire entre ingénieurs mécaniciens et électriciens qui participent à l'élaboration de centrales hydroélectriques. 